\title{
EL PESIMISMO DEFENSIVO Y EL SÍNDROME DEL IMPOSTOR: ANÁLISIS DE SUS COMPONENTES AFECTIVOS Y COGNITIVOS
}

\author{
EVARISTO FERNÁNDEZ y JOSÉ BERMÚDEZ \\ Facultad de Psicología, Universidad Nacional de Educación a Distancia
}

(Recibido el 5 de diciembre de 1998)

\begin{abstract}
En este trabajo se lleva a cabo un estudio comparativo de las características asociadas al Pesimismo defensivo (PD) y el Síndrome del Impostor (SI), dos patrones de comportamiento que tienen en común la existencia de dudas acerca de la propia habilidad, miedo al fracaso y mantenimiento de unas bajas expectativas de resultado, todo ello a pesar de una importante historia de éxitos. No obstante, al mismo tiempo pensamos que estos dos constructos pueden diferenciarse en el grado en que manifiestan estas y otras respuestas, creencias y actitudes. Para explorar esta posibilidad, realizamos una serie de análisis de correlaciones y de regresión jerárquica y múltiple sobre diversas variables de carácter afectivo y cognitivo. Los resultados revelan un patrón claramente diferenciado para ambos constructos. Concretamente, el PD parece caracterizarse por ansiedad de prueba, (bajo) optimismo, (baja) autoestima académica y pesimismo, mientras que el SI estaría caracterizado por confusión en el autoconcepto, (baja) autoestima social y global, (baja) autoeficacia, externalismo, autoconciencia y ansiedad social. En la discusión resaltamos el carácter más desadaptativo del SI, en comparación con el PD, y su naturaleza más de tipo social o interpersonal. Finalmente, abordamos la cuestión de la direccionalidad de los efectos hallados, sugiriendo la existencia de una continua retroalimentación entre ambos patrones de comportamiento y sus componentes.
\end{abstract}

Palabras clave: Pesimismo defensivo, síndrome del impostor, expectativas, incompetencia percibida, autoestima, protección.

The defensive pessimism and the impostor syndrome: an analysis of their affective and cognitive components

This work undertakes a comparative study of the characteristics associated to the Defensive pessimism (DP) and the Impostor syndrome (IS), two patterns of behavior characterized by self-doubts, fear of failure, and low outcome expectancies, in spite of a history of success. At the same time, we think that these two constructs can be different in the degree that show these and other responses, beliefs, and attitudes. To check this hypothesis, a series of correlational and hierarchical and multiple regression analyses on several affective and cognitive variables were conducted. The results showed a different pattern for each construct. Specifically, DP is better defined by test anxiety (worry), (low) optimism, (low) academic self-esteem, and pessimism, whereas self-concept confusion, (low) social and global self-esteem, trait anxiety, (low) self-efficacy, externalism, self-consciousness, and social anxiety are more important in IS. In the discussion we emphasize that, compared with DP, IS shows a more inadaptative character, and is more social or interpersonal in nature. Finally, we consider the effects directionality question, and we suggest a continuous feedback among these behavior patterns and their components.

Key words: Defensive pessimism, impostor syndrome, low expectancies, perceived incompetence, self-esteem, protection.

\section{INTRODUCCIÓN}

\section{La existencia de unas expectativas ne-}

Correspondencia: Facultad de Psicología, UNED, Dpto. Personalidad, Evaluación y Tratamientos Psicológicos, Ciuded Universitaria s/n., 28040 Madrid. Tfno.: 91398 6517, Fax: 91398 6298. Correoe: efernandez@bec.uned.es gativas y de dudas ante el afrontamiento futuro de una situación de logro en personas con una importante experiencia de éxitos, ha llamado la atención de algunos investigadores en los últimos años (Clance e Imes, 1978; Clance y O'Toole, 1987; Harvey, 1981; Kolligian y Sternberg, 
1991; Norem y Cantor, 1986a; 1986b; Norem y Illingworth, 1993; Showers y Rubens, 1990; Cozzarelli y Major, 1990). Gracias a este esfuerzo investigador se han identificado dos patrones comportamentales, el Síndrome del Impostor (SI) (Clance, 1985) y el Pesimismo Defensivo (PD) (Norem y Cantor, 1986a).

El síndrome del impostor ha sido definido como un sentimiento intenso de falsedad o falta de autenticidad con respecto a la autoimagen de competencia, experimentado por personas con una apreciable historia de éxitos (Clance, 1985). A pesar de estos logros, los denominados impostores manifiestan importantes dudas acerca de sus habilidades, $y$ creen que éstas son continua e injustificadamente sobreestimadas por los demás. De esta manera, no se creen merecedores de los éxitos que obtienen, y se preocupan de que los demás puedan descubrir en cualquier momento que no son tan inteligentes como parecen; en este sentido, el éxito les hace sentirse como unos farsantes (Clance, 1985). Como consecuencia de su miedo y ansiedad ante la posibilidad de fracasar, y así parecer ante los demás como unos incompetentes, los impostores se esfuerzan para evitar un posible fracaso que "pueda delatarles» (Clance e Imes, 1978; Clance y O'Toole, 1987).

Como consecuencia del patrón atribucional mostrado por los impostores ante los éxitos, caracterizado por una externalización del éxito y una internalización del fracaso, estas personas suelen experimentar un sentimiento de culpabilidad ante aquel resultado, lo que contribuye al desarrollo de los sentimientos de falsedad mencionados previamente, así como al temor de que los demás descubran que en realidad esas habilidades que les asignan no son las responsables de los resultados que obtienen. Clance (1985), por otra parte, observa que el éxito repetido no debilita este tipo de sentimientos en los impostores, ni estimula la creencia en una alta habilidad. Asimismo, Clance y O’Toole (1987) señalan que estas personas muestran un gran ingenio a la hora de negar la evidencia externa de habilidad y de desacreditar las valoraciones positivas procedentes de los demás.

Desde el punto de vista clínico el síndrome del impostor se ha asociado con diversos tipos de síntomas como la ansiedad generalizada, depresión, falta de autoconfianza, baja autoestima y frustración relacionada con la imposibilidad de cumplir con los altos estándares autoimpuestos (Chrisman, Pieper, Clance, Holland y Glickauf-Hughes, 1995; Clance e Imes, 1978; Matthews y Clance, 1985).

Por su parte, el pesimismo defensivo es conceptualizado como una estrategia cognitiva de dominio específico (de logro, social), consistente en adoptar unas bajas expectativas y metas ante la existencia de una situación futura a afrontar, a pesar de que las personas que recurren a ella presentan una clara historia de éxitos, algo que reconocen como cierto. Estas personas se sienten inicialmente ansiosas y fuera de control, y focalizan su atención en las dificultades y resultados negativos posibles, incluso aunque parezcan poco probables (Norem y Cantor, 1986a).

La evidencia demuestra que estas expectativas no se convierten en profecías autocumplidas, ni llevan a una retirada del esfuerzo, como sí parece ocurrir en los pesimistas puros o disposicionales (no defensivos). En lugar de eso, los pesimistas defensivos invierten una gran cantidad de esfuerzo en tareas que ellos valoran como importantes (Cantor, Norem, Niedenthal, Langston y Brower, 1987; Norem y Cantor, 1986b; Norem y Cantor, 1990). Además, el nivel de rendimiento alcanzado por ellos es comparable al de los sujetos que utilizan la estrategia contraria, esto es, el optimismo. Asimismo, los pesimistas defensivos parecen encontrarse protegidos gracias a la utilización 
de sus estrategias, pues no se sienten menos satisfechos que los optimistas después de un fracaso, ni recurren a juicios atribucionales que les permitirían negar la responsabilidad sobre el mal rendimiento y así proteger su autoestima (Fernández, 1999; Norem y Cantor, 1986a).

Desde el punto de vista clínico se ha observado que los pesimistas defensivos presentan a largo plazo, en comparación con los optimistas, mayores niveles de sintomatología informada (preocupación, insomnio, desesperanza), nivel de estrés $\mathrm{y}$ de insatisfacción vital (Cantor et al., 1987), y un mayor nivel de depresión, aunque menor que el de los pesimistas puros (no defensivos) (Hummer, Dember, Melton y Schefft, 1992). De igual manera, se ha encontrado que el pesimismo defensivo correlaciona negativamente con autoestima y autoeficacia generalizada, haciéndolo positivamente con ansiedad y externalismo (en este último caso en menor medida que el pesimismo puro) (Fernández y Bermúdez, 1999).

Una primera conclusión que puede sacarse a partir de la descripción de ambos patrones de comportamiento se refiere a la existencia de importantes similitudes entre ellos, tanto desde el punto de vista del dinamismo psicológico que los caracteriza como de sus consecuencias adaptativas, siendo esta la razón que llevó a Cozzarelli y Major (1990) a estudiarlos conjuntamente. Por un lado, tanto los impostores como los pesimistas defensivos experimentan miedo al fracaso, ansiedad y duda acerca de las propias posibilidades de lograr buenos resultados, y se fijan unas bajas expectativas ante cada nueva situación a afrontar. Esta valoración inicial de la situación y de sí mismos conduce a ambos grupos a invertir un gran esfuerzo para tratar de evitar el posible fracaso.

Por otro lado, una segunda característica que definiría a ambos grupos de sujetos está relacionada con las repercusiones que puede tener para la cualidad del autoconcepto el hecho de, por un lado, tener una historia personal de éxitos y, por otro, mantener continuas dudas acerca de la propia competencia de cara al afrontamiento exitoso de futuras situaciones. Específicamente, la consecuencia esperable de esta contradicción puede ser el mantenimiento de una baja claridad o certeza en el autoconcepto, que englobaría tanto aspectos positivos (relacionados con los éxitos pasados) como negativos (relacionados con el miedo al fracaso y las bajas expectativas de éxito). Esta posibilidad, que sí ha sido contemplada por algunos autores en el caso del pesimismo defensivo (Fernández, 1999, exp. 2; Harlow y Cantor, 1995), no ha sido objeto de análisis aún en el del síndrome del impostor. Uno de los objetivos del presente trabajo consiste en aportar datos sobre esta cuestión.

Por otro lado, junto a estas similitudes, puede sugerirse a partir del único estudio existente sobre ambos constructos (Cozzareli y Major, 1990), la existencia de diferencias en el grado en que PD y SI contienen las características descritas anteriormente; diferencias que podrían condicionar el hecho de que el SI resulte ser un patrón de comportamiento más desadaptativo. Adicionalmente, estos constructos no han sido comparados anteriormente con respecto a otras variables básicas para su comprensión, como es el caso del optimismo-pesimismo, la autoestima, el lugar de control y la autoconciencia. La primera de ellas (el optimismo-pesimismo) puede estar presente en diverso grado en ambos constructos, en donde cobra especial relevancia la naturaleza de las expectativas que desarrollan acerca de los resultados futuros. Asimismo, la (baja) autoestima parece jugar un papel relevante en diversas reacciones manifestadas por pesimistas defensivos (Harlow y Cantor, 1995) e impostores (Langford y Clance, 1993). 
Por lo que respecta a las expectativas sobre la controlabilidad de los resultados existe evidencia directa o indirecta de que unos y otros sujetos perciben cierto grado de incontrolabilidad sobre el ambiente, lo que parece afectar a sus reacciones iniciales. Finalmente, la autoconciencia puede que esté caracterizando a ambos constructos. Por un lado, Fernández y Bermúdez (1999) han informado de la existencia de asociación positiva significativa, aunque moderada, entre esta variable y el PD. Por otro, a partir de otro estudio efectuado por estos autores (Fernández y Bermúdez, en prensa) puede deducirse que la autoconciencia tenga también su relevancia en el SI, pues este se caracteriza por un interés por evitar una impresión desfavorable en los demás (autoobservación protectora), actitud que implica, necesariamente, ser consciente de los propios sentimientos y conductas, y de cómo afectan a otras personas. En el presente trabajo trataremos de aportar evidencia más directa acerca del grado en que el PD y el SI comparten todas estas actitudes y creencias, $\mathrm{o}$, por el contrario, difieren en ellas.

Así pues, el presente estudio se plantea como objetivo global la exploración de las posibles relaciones existentes entre el síndrome del impostor y el pesimismo defensivo, por un lado, y terceras variables de naturaleza cognitiva y afectiva, por otro, en un intento de profundizar tanto en las diferencias como en las semejanzas que puedan existir entre ambos constructos. No obstante, para cumplir adecuadamente con este objetivo es necesario proceder de manera especial. Hay que tener en cuenta que el PD y el SI se solapan en cierto grado desde el punto de vista conceptual, lo que se ha traducido, en el plano empírico, en correlaciones moderadamente altas entre ellos ( $r=0,52$, en el estudio de Cozzarelli y Major, 1990; y $r=0,57$ en el estudio de Fernández y Bermúdez, en prensa). Estos índices de asociación reflejan un grado apreciable de varianza compartida por ambos constructos, aunque, paralelamente, ponen de manifiesto cierto grado de independencia, que hace aconsejable emprender un estudio de sus cualidades diferenciadoras específicas. Por esta razón, y siguiendo los pasos de un trabajo anterior (Fernández y Bermúdez, en prensa), procederemos a analizar, en un primer momento, las características asociadas a ambos constructos en términos absolutos, para pasar posteriormente a contemplar por separado cada uno de ellos previo control estadístico de la influencia ejercida por el otro. Sólo de esta manera podremos obtener una comprensión adecuada de la verdadera naturaleza del PD y el SI.

\section{MÉTODO}

\section{Sujetos y procedimiento}

Participaron 140 estudiantes del primer ciclo de Psicología de la UNED, de los cuales 65 eran hombres y 75 mujeres. El rango de edad es de 18 a 42 años $(M=$ 27,65; DT $=7,14$ ).

Se entregaron en clase distintos inventarios con instrucciones para que los complementaran en sus casas y los enviaran por correo a la Facultad. El orden de cumplimentación fue el que aparece a continuación en la descripción de los instrumentos.

\section{Instrumentos}

1. Optimism-Pessimism Questionnaire $(O P Q)($ Norem y Cantor, 1986a). Medida de optimismo y pesimismo defensivo referidos a situaciones académicas. Originalmente, este inventario consta de 9 items, siendo 4 de ellos de contenido optimista; 4 de conte- 
nido pesimista, y uno referido al rendimiento pasado. La escala de respuesta es de tipo Likert de 11 puntos, donde 1= En total desacuerdo y 11= Totalmente de acuerdo. Para el presente estudio hemos utilizado la subescala de pesimismo, junto con el ítem sobre rendimiento pasado. Este último permite discriminar entre sujetos pesimistas puros o no defensivos, que reconocen haber rendido pobremente en el pasado en situaciones académicas (puntuación inferior a 6 en la escala de respuesta), y defensivos, que informan de un alto rendimiento pasado (puntuación de 7 o más). Nosotros tendremos en cuenta este último criterio en los diferentes análisis. Por otra parte, a partir de un estudio anterior en torno a este inventario (Fernández y Bermúdez, 1999), hemos añadido un ítem de contenido pesimista, que es una reformulación de otro ítem (también pesimista), destinado a mejorar su calidad en el conjunto de la escala. Fernández y Bermúdez (1999) informan de una consistencia interna aceptable para esta subescala (alfa= 0,76). Ejemplos de items son los siguientes: «Al enfrentarme a situaciones académicas espero lo peor, incluso aunque sepa que probablemente lo haré bien"; "Suelo dudar tanto de poder rendir bien ante una prueba que es un alivio cuando la supero satisfactoriamente»).

2. Impostor Phenomenon (IP; Clance y O'Toole, 1988). Medida del Síndrome del Impostor. Contiene 20 items destinados a evaluar pensamientos y sentimientos relacionados con dudas y preocupaciones acerca de la propia capacidad, preocupación por haber logrado cosas por casualidad o por error, más que por la propia habilidad y falta de reconocimiento del propio mérito en la obtención de los éxitos pasados. Dos ejemplos de items son los siguientes: "Temo que personas importantes para mí puedan descubrir que no soy tan capaz como ellas piensan que soy»; "Si recibo halagos o reconocimiento por algo que he logrado, tiendo a quitar importancia a lo que he hecho». La escala de respuesta es de tipo Likert de 5 puntos, donde $1=$ Totalmente en desacuerdo y $5=$ Totalmente de acuerdo.

3. State-Trait Anxiety Inventory, escala de rasgo (STAI-R) (Spielberger, Gorsuch y Lushene, 1970). Contiene 40 items distribuidos en dos escalas, una de ansiedad-estado y otra de ansiedad-rasgo, que es la que utilizaremos aquí, con 20 items cada una. Se evalúa con una escala de respuesta tipo Likert de 4 puntos, donde 1= Casi nunca y 4= Casi siempre.

4. Personal and Academic Self-Concept Inventory (PASCI) (Fleming y Whalen, 1990). Se trata de un inventario de autoconcepto y autoestima de 45 items. Contiene 7 subescalas: autoestima global, aceptación social, habilidad académica, habilidad verbal, habilidad para las matemáticas, apariencia física, habilidad física, aceptación parental y ansiedad social. Aquí utilizaremos sólo 3 de ellas, con un total de 15 items: autoestima global, autoestima académica y autoestima social. En la versión original cada ítem está formulado como una pregunta, y la escala de respuesta es de tipo Likert de 7 puntos (1= Prácticamente nunca; 7= Muy frecuente). Para este estudio hemos modificado la manera de presentar los items, que aparecen como una afirmación, y la escala de respuesta conserva la amplitud de 7 puntos, aunque los extremos se denominan de otra manera ( $1=\mathrm{No}$ en absoluto, $7=$ Sí totalmente). 
5. Self-Concept clarity (SCC) (Campbell, Trapnell, Heine, Katz, Lavallee y Lehman, 1996). Medida de claridad en el autoconcepto, de 12 items. Incluye tres componentes que saturan en un único factor: consistencia interna percibida, estabilidad temporal de las creencias acerca de uno mismo y certeza sobre las autodescripciones. La escala de respuesta es de tipo Likert de 5 puntos ( $1=$ En total desacuerdo; $5=$ Totalmente de acuerdo).

6. Life Orientation Test (LOT) (Scheier y Carver, 1985a; Scheier, Carver y Bridges, 1994). Medida de optimismo disposicional que estuvo formada originalmente por 12 items ( 8 efectivos y 4 de control). De los 8 items válidos, 4 estaban formulados positivamente (optimismo) y 4 negativamente (pesimismo). Posteriormente sufrí una revisión (1994), en la que de estos 8 items sólo quedaron 5 , añadiendo uno más. Nosotros utilizaremos esta última versión, que contiene 3 items de contenido optimista y 3 de contenido pesimista. La escala de respuesta para cada uno de ellos es de tipo Likert de 5 puntos, donde $1=$ Muy en desacuerdo y $5=$ Totalmente de acuerdo.

7. Locus of Control of Behavior (LCB) (Craig, Franklin y Andrews, 1984). Medida del lugar de control de la conducta, de 17 items, que conforman un factor único, Externalismo. Se evalúa a través de una escala de respuesta tipo Likert de 6 puntos, donde $1=$ Totalmente en desacuerdo y 6=Totalmente de acuerdo.

8. Self-Efficacy Scale (SES) (Sherer et al. 1982). Medida de autoeficacia generalizada de 24 items, de los cuales 17 son efectivos y 7 son de control. Se evalúan en una escala de respuesta de 5 puntos ( $1=$ Totalmente en desacuerdo, $5=$ Totalmente de acuerdo).
9. Test Anxiety Inventory (TAI) (Spielberger, 1980). El TAI es una inventario de ansiedad de prueba de amplia utilización en la literatura sobre ansiedad y ejecución en contextos de logro. Contiene dos subescalas, Preocupación (Worry) y Emocionalidad (Emotionality), y una puntuación global. Los 20 items de que consta se evalúan por medio de una escala de respuesta tipo Likert de 4 puntos (1=Casi nunca, 4=Casi siempre).

10. Self-Consciousness Scale (SCS) (Scheier y Carver, 1985b). Medida de autoconciencia, revisada a partir del inventario original de Fenigstein, Scheier y Buss (1975). Consta de tres escalas: autoconciencia privada, pública y ansiedad social. Contiene 22 items que se puntúan en una escala de respuesta tipo Likert de 5 puntos, donde $1=$ Nada aplicable y $5=$ Totalmente aplicable.

\section{RESULTADOS}

La consecución de los objetivos previamente descritos requiere llevar a cabo la exploración de las asociaciones entre el PD y el SI, por un lado, y de ambos con las variables de carácter cognitivo y afectivo evaluadas, por otro. Este análisis se llevará a cabo en dos fases. Como ya hemos adelantado anteriormente, en primer lugar calcularemos las correlaciones absolutas existentes entre las variables mencionadas, para pasar, en un segundo momento, a contemplar la contribución relativa de todas ellas por medio del control estadístico del efecto del SI sobre el $\mathrm{PD}$, por un lado, y del efecto de este último sobre el primero, por otro. Dentro de esta segunda fase de análisis, procederemos, adicionalmente, a explorar aquellas variables que integrarían las ecuaciones predictivas de ambos constructos. 


\section{Análisis de las correlaciones absolutas entre variables}

En la Tabla 1 aparecen los datos referentes al primer tipo de análisis referido, esto es, al análisis de las asociaciones absolutas entre variables. En primer lugar, un primer dato que merece resaltarse es la correlación moderadamente alta existente entre el PD y el SI, reflejando un solapamiento apreciable entre ambas variables, si bien subsiste un grado de independencia suficiente como para que resulte aconsejable estudiarlas separadamente.

Por otra parte, y de manera coherente con la asociación descrita entre el PD y el SI, un examen de la Tabla 1 revela una notable semejanza en los diversos correlatos analizados, con algunas excepciones. Desde el punto de vista afectivo, se observa que tanto el PD como el SI correlacionan positiva y significativamente con ansiedad rasgo y ansiedad de prueba, y negativamente con autoestima. Con respecto a las medidas de tipo más cognitivo puede observarse que los dos cons- tructos correlacionan negativamente con claridad del autoconcepto, autoeficacia y optimismo, mientras que lo hacen positivamente con pesimismo y externalismo.

Con respecto a la autoconciencia, claramente se observa que el SI, pero no el PD, correlaciona significativamente tanto con la modalidad privada como con la pública, mientras que ambos constructos se asocian positivamente con ansiedad social.

\section{Análisis de las asociaciones relativas entre variables: control del efecto diferencial del PD y el SI}

Un segundo momento en el análisis de los datos consistió en la exploración de la contribución relativa del PD y el SI, mediante la separación de aquella parte de la varianza común a ambos, algo que viene justificado por la importante asociación existente entre ellos, como hemos visto anteriormente. Procediendo de esta manera podremos demostrar qué constructo es responsable en último término

Tabla 1. Correlaciones entre el pesimismo defensivo y el síndrome del impostor con variables de carácter afectivo y cognitivo

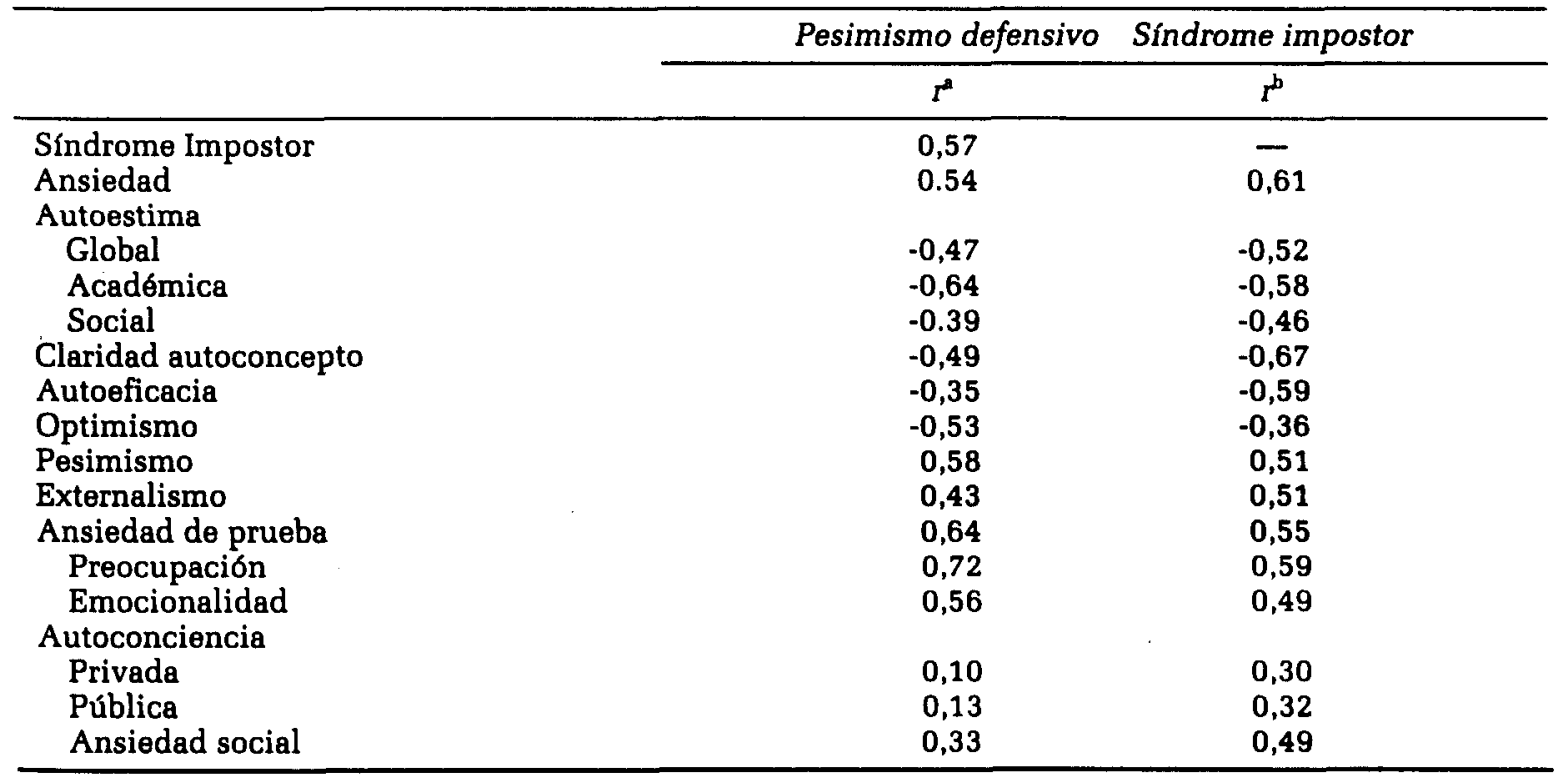

Nota: $\mathrm{N}=118$. "Todos los coeficientes son significativos $(p<0,001)$, con la excepción de los correspondientes a la autoconciencia pública y privada, que no lo son en absoluto. ${ }^{b}$ Todos los coeficientes son significativos $(p \leq 0,001)$. 
de las asociaciones descritas más arriba, así como conocer el grado en que cada una de ellas se mantiene, aumenta o disminuye cuando se contempla a cada constructo de manera independiente.

Realizamos una serie de análisis de regresión jerárquica (Enter) para el PD y el SI por separado, sobre cada una de las variables cognitivas y afectivas estudiadas. Específicamente, en el caso del PD introdujimos en primer lugar en la ecuación de regresión la variable SI, con lo cual su efecto sobre aquél quedaba controlado cuando adicionalmente añadíamos, una por una, el resto de las variables mencionadas. Por el contrario, cuando el análisis de regresión se realizaba para el SI, procedíamos de la misma forma, pero esta vez controlando el efecto del PD, que era introducido en la ecuación en primer lugar.

Los resultados correspondientes a estos análisis aparecen en la Tabla 2. Puede observarse que tuvieron lugar una serie de cambios en el patrón de asociaciones con respecto al manifestado en los análisis previos. En primer lugar, en el caso del $\mathrm{PD}$, que en términos absolutos correlacionaba negativamente con claridad del autoconcepto y autoeficacia, la contribución relativa de estas variables a la varianza total no era significativa cuando manteníamos constante el efecto del SI, mientras que la varianza adicional explicada para el SI por la claridad y la autoeficacia cuando controlamos el efecto del PD sí que resultaba significativa. De esta manera, la tendencia a una falta de claridad del autoconcepto y a una baja autoeficacia parece ser algo específico del SI, no del PD.

Por otra parte, la ansiedad social únicamente explicaba una porción de varianza adicional significativa en el caso del SI, cuando en los análisis previos aquella variable se asociaba significativamente con ambos constructos.

Con respecto a la autoestima social, cabe decir que sólo en el caso del SI añadía poder explicativo, mientras que en

Tabla 2. Análisis de regresión jerárquica (Enter) del pesimismo defensivo (manteniendo constante el efecto del síndrome del impostor) y del síndrome del impostor (manteniendo constante el efecto del pesimismo defensivo) sobre variables de naturaleza afectiva y cognitiva

\begin{tabular}{|c|c|c|c|c|c|c|}
\hline & \multicolumn{3}{|c|}{ Pesimismo defensivo } & \multicolumn{3}{|c|}{ Sindrome impostor } \\
\hline & $R^{2}$ camb. $^{a}$ & sig. $F$ & $r p$ & $R^{2} c a m b .^{b}$ & sig. $F$ & $r p$ \\
\hline Ansiedad & 0,05 & 0,001 & 0,29 & 0,14 & 0,0000 & 0,49 \\
\hline \multicolumn{7}{|l|}{ Autoestima } \\
\hline Global & 0,04 & 0,006 & $-0,25$ & 0,09 & 0,0002 & $-0,34$ \\
\hline Académica & 0,13 & 0,0000 & $-0,44$ & 0,07 & 0,0001 & $-0,44$ \\
\hline Social & 0,02 & 0,074 & $-0,16$ & 0,09 & 0,0003 & $-0,16$ \\
\hline Claridad autoconcepto & 0,006 & N.S. & $-0,11$ & 0,21 & 0,0000 & $-0,53$ \\
\hline Autoefícacia & 0,001 & N.S. & 0,01 & 0,23 & 0,0000 & $-0,51$ \\
\hline Optimismo & 0,16 & 0,0000 & $-0,42$ & 0,004 & N.S. & $-0,08$ \\
\hline Pesimismo & 0,12 & 0,0000 & 0,40 & 0,05 & 0,002 & 0,27 \\
\hline Externalismo & 0,03 & 0,03 & 0,20 & 0,10 & 0,0001 & 0,36 \\
\hline Ansiedad de prueba & 0,16 & 0,0000 & 0,48 & 0,05 & 0,001 & 0,30 \\
\hline Preocupación & 0,22 & 0,0000 & 0,58 & 0,048 & 0,0004 & 0,32 \\
\hline Emocionalidad & 0,12 & 0,0000 & 0,39 & 0,04 & 0,007 & 0,24 \\
\hline \multicolumn{7}{|l|}{ Autoconciencia } \\
\hline Privada & 0,005 & N.S. & $-0,08$ & 0,083 & 0,0014 & 0,29 \\
\hline Pública & 0,003 & N.S. & $-0,06$ & 0,09 & 0,0009 & 0,30 \\
\hline Ansiedad social & 0,003 & N.S. & 0,06 & 0,14 & 0,0000 & 0,39 \\
\hline
\end{tabular}

Nota: $\mathrm{N}=118 ; \mathrm{rp}=$ correlación parcial. ${ }^{2} \mathrm{R}^{2}$ cambio: proporción de varianza adicional explicada por cada variable, con respecto a la que ya explica el Síndrome del Impostor, que es forzado a entrar en primer lugar en la ecuación. ${ }^{b} R^{2}$ cambio: proporción de varianza adicional explicada por cada variable, con respecto a la que ya explica el Pesimismo Defensivo, que es forzado a entrar en primer lugar en la ecuación. 
relación al PD la varianza adicional explicada era sólo marginalmente significativa. En el caso del optimismo ocurría lo contrario. Cuando se controlaba el efecto del PD la contribución de aquella variable a la varianza total del SI era prácticamente inapreciable, mientras que la correspondiente al PD cuando se mantenía constante el SI sí era importante. En el caso del pesimismo, y también en el de ambos componentes de la ansiedad de prueba (principalmente la preocupación) la cantidad de varianza explicada tanto del PD como del SI era significativa, produciéndose en esta ocasión sólo diferencias en la magnitud de la misma, que era mayor en el caso del PD. La tendencia contraria existía con respecto a la ansiedad rasgo y externalismo, con una contribución relativa mayor en el caso del SI.

\section{Análisis de regresión múltiple para el PD y el SI}

Un tercer y último tipo de análisis se planteaba la exploración de los mejores predictores de cada uno de los constructos estudiados, el PD y el SI. Realizamos sen- dos análisis de regresión múltiple (Stepwise) sobre el resto de variables de tipo cognitivo y afectivo. El criterio de selección de estas últimas consistió en tomar a aquéllas que en el segundo tipo de análisis realizado anteriormente (de asociaciones relativas) contribuían significativamente a la varianza. Específicamente, en los análisis efectuados para el PD, las variables consideradas fueron ansiedad, autoestima global y académica, optimismo, pesimismo, externalismo, y preocupación y emocionalidad, correspondientes a la ansiedad de prueba. Por su parte, los análisis realizados para el SI se basaron en todas las variables salvo el optimismo.

Adicionalmente, para estos análisis volvimos a tener en cuenta el hecho de que el PD y el SI se solapan en cierto grado. Así pues, previamente al análisis destinado a obtener la mejor ecuación de regresión para cada constructo, forzamos la entrada en la misma (Enter) del SI, cuando los análisis se efectuaban para el PD, o del PD, cuando se realizaban para el SI. De esta manera, volvíamos a mantener constantes los efectos correspondientes a cada constructo.

Tabla 3. Análisis de regresión múltiple (Stepwise) del pesimismo defensivo (manteniendo constante el efecto del síndrome del impostor) y del síndrome del impostor (manteniendo constante el efecto del pesimismo defensivo) sobre variables de naturaleza afectiva y cognitiva

\begin{tabular}{|c|c|c|c|c|c|c|c|}
\hline \multirow[b]{2}{*}{ Variables } & \multicolumn{7}{|c|}{ Pesimismo defensivo } \\
\hline & $R$ & $R^{2}$ aj. & $F$ & $R^{2}$ camb. & $F$ camb. & $\beta$ & $r p$ \\
\hline $\begin{array}{l}\text { Enter: } \\
\text { Sịndrome del Impostor Stepwise: } \\
\text { Preocupación } \\
\text { Optimismo } \\
\text { Autoestima académica }\end{array}$ & $\begin{array}{l}0,57 \\
0,74 \\
0,77 \\
0,78\end{array}$ & $\begin{array}{l}0,32 \\
0,54 \\
0,58 \\
0,60\end{array}$ & $\begin{array}{l}56,2^{a} \\
71,4^{a} \\
56,9^{a} \\
45,3^{4}\end{array}$ & $\begin{array}{l}0,23 \\
0,046 \\
0,016\end{array}$ & $\begin{array}{c}58,8^{\mathrm{a}} \\
13,1^{\mathrm{a}} \\
4,8^{\mathrm{c}}\end{array}$ & $\begin{array}{r}0,13 \\
0,41 \\
-0,23 \\
-0,18\end{array}$ & $\begin{array}{r}0,16 \\
0,41 \\
-0,31 \\
-0,20\end{array}$ \\
\hline \multirow{2}{*}{ Autoestima académica } & \multicolumn{7}{|c|}{ Sindrome del impostor } \\
\hline & $R$ & $R^{2}$ aj. & $F$ & $R^{2}$ camb. & $F$ camb. & $\beta$ & $r p$ \\
\hline $\begin{array}{l}\text { Enter: } \\
\text { Pesimismo defensivo } \\
\text { Stepwise: } \\
\text { Claridad autoconcepto } \\
\text { Autoestima social } \\
\text { Preocupación }\end{array}$ & $\begin{array}{l}0,75 \\
0,77 \\
0,79\end{array}$ & $\begin{array}{l}0,56 \\
0,59 \\
0,61\end{array}$ & $\begin{array}{l}59,6^{\circ} \\
44,7^{\circ} \\
36,6^{4}\end{array}$ & $\begin{array}{l}0,24 \\
0,03 \\
0,02\end{array}$ & $\begin{array}{r}59,9^{a} \\
6,9^{\mathrm{b}} \\
5,1^{\mathrm{c}}\end{array}$ & $\begin{array}{r}-0,43 \\
-0,27 \\
0,22\end{array}$ & $\begin{array}{r}-0,49 \\
-0,30 \\
0,24\end{array}$ \\
\hline
\end{tabular}

Nota: $\mathrm{N}=118 ; \mathrm{R}^{2}$ aj.= proporción de varianza explicada ajustada; $\mathrm{R}^{2}$ camb.= proporción de varianza adicional explicada; $B=$ coeficiente de regresión estandarizado; $r p=$ correlación parcial. $" p<0,001 .{ }^{b} p<$ $0,01 .{ }^{c} p<0,05$. 
En la Tabla 3 aparecen representados los modelos finales de regresión para el PD y el SI. En ambos casos fueron tres las variables que mejor los predecían, logrando explicar porcentajes de varianza importantes $(60 \%$ de la correspondiente al PD y el $61 \%$ de la existente en el SI).

En el PD la primera variable en entrar fue el componente de preocupación de la ansiedad de prueba, que predecía positivamente el criterio $(B=0,41)$. En segundo lugar entró el optimismo, prediciendo en sentido negativo $(\beta=-0,23)$. Finalmente, la autoestima académica se asociaba también negativamente con el $P D(B=-0,18)$.

Con respecto al SI, su principal predictor fue la claridad del autoconcepto, en sentido negativo $(B=-0,48)$. Posteriormente estaba la autoestima social, prediciendo también en sentido negativo $(B=$ $0,25), y$, finalmente, la última variable en entrar en la ecuación fue la preocupación, prediciendo en sentido positivo $(B=0,24)$.

En conclusión, tanto en el PD como en el SI resulta relevante la preocupación acerca de la posibilidad de fracasar, aunque ésta parece tener más relevancia relativa en el primero de ellos. Con respecto a la autoestima, es el contenido académico del autoconcepto lo esencial en el PD, mientras que el aspecto social predomina en el SI. Por último, un bajo optimismo es específico del PD, mientras que una baja claridad en el autoconcepto lo es del SI.

\section{DISCUSIÓN}

El presente estudio tenía por objeto la exploración de las posibles similitudes y diferencias existentes entre dos constructos que presentan una importante similitud desde el punto de vista conceptual (bajas expectativas, miedo al fracaso, historia de éxitos, etc.).

A partir del análisis del patrón de asociaciones existente con otras variables de tipo cognitivo y afectivo, y de los mode- los predictivos hallados para cada uno de ellos, pudimos ir desentrañando, paso a paso, la cualidades tanto diferenciales como comunes existentes en cada constructo. El análisis absoluto de estas asociaciones puso de manifiesto principalmente, y salvo raras excepciones, la existencia de importantes similitudes en el tipo de componentes cognitivos y afectivos que los caracterizan, semejanzas que venían justificadas por el solapamiento apreciable existente entre el PD y el SI. Posteriormente, cuando pasamos a considerar un análisis más específico de los mismos (asociaciones relativas) comenzaron a ponerse de manifiesto una serie diferencias que permitían constatar la existencia de cualidades específicas a cada uno de ellos. Pasemos a contemplar las implicaciones que pueden derivarse de todo ello.

En primer lugar, en ambos constructos resultaba esencial el componente cognitivo de la ansiedad de prueba (preocupación), y también el emocional. Tanto desde la literatura del SI como de la correspondiente al PD se consideran a la ansiedad inicial acerca de los posibles resultados, las dudas sobre la propia habilidad y el consiguiente estado de bloqueo emocional, como elementos activadores de estrategias de preparación ante la posibilidad de fracasar. En el caso del SI, la reacción ante ese estado inicial consiste en invertir una gran cantidad de esfuerzo, que iría destinado a evitar el fracaso con el fin de impedir, al mismo tiempo, la desaprobación de los demás (Clance y O'Toole, 1988; Fernández y Bermúdez, en prensa).

En los pesimistas defensivos, por otra parte, esta reacción consiste en bajar las expectativas de éxito (Norem y Cantor, 1986a), las metas, y en devaluar los resultados futuros (Fernández, 1999) con un propósito claramente protector, que permitiría el control de la ansiedad y el aumento del control y la motivación 
(esfuerzo), hasta ese momento inhibida por la existencia de un conflicto inicial entre el motivo de logro y el miedo al fracaso.

No obstante, a pesar de la relevancia de la preocupación en ambos constructos, todos los análisis revelaban que aquélla estaba más presente en el PD, contrariamente a lo señalado inicialmente por Cozzarelli y Major (1990). Estos autores defienden que son los sujetos impostores los que pueden manifestar un mayor miedo al fracaso (ansiedad de prueba, preocupación) que los pesimistas defensivos, lo que explicaría el carácter no protector de unas bajas expectativas en aquellos sujetos y, por tanto, su mayor desadaptación, en comparación con los pesimistas defensivos, en donde esta protección sí está presente, como ya hemos señalado. Por el contrario, nuestros datos indican que la contribución relativa de la ansiedad de prueba es mayor en el PD que en el SI, lo que sugiere que el miedo al fracaso en una situación de logro, al menos, puede que sea mayor en los pesimistas defensivos que en los impostores. De esta manera, la explicación que ofrecen estos autores de la ausencia de funcionalidad protectora de unas bajas expectativas en los sujetos impostores, en términos de un mayor miedo al fracaso, no estaría justificada por nuestros datos. Otro tipo de factores pueden resultar relevantes en este sentido. Específicamente, Fernández y Bermúdez (en prensa) encuentran datos a favor de la existencia de un vínculo entre el estilo atribucional desadaptativo de los impostores y la ausencia de protección afectiva ante un futuro fracaso.

Por contraposición, la ansiedad contemplada como algo más transituacional o generalizado (y no solo referida a una situación de prueba) parecía estar más presente en el SI que en el PD, como sugerían los datos correspondientes al análisis de las asociaciones relativas entre variables.

La existencia de diferencias entre el SI y el PD en cuanto a la manifestación de distintos tipos de ansiedad resulta de gran interés teórico, pues abre nuevas perspectivas de cara a la conceptualización de ambos constructos. En lo que respecta a la ansiedad de prueba, estas diferencias son sorprendentes, si tenemos en cuenta que tanto en el SI como en el PD está muy presente la experiencia de ansiedad y miedo al fracaso en un contexto de logro, en donde tan frecuentes son las situaciones de evaluación de la propia competencia. Nosotros pensamos, de manera consistente con lo encontrado por Topping (1983) y con lo sugerido por Langford y Clance (1993), que es muy probable que las personas con sentimientos de impostor experimenten ansiedad en la mayoría de las situaciones, entre las cuales, añadimos nosotros, pueden encontrarse una situación de prueba, aunque no tenga por qué reducirse a ella y/o la experiencia de ansiedad sea tan intensa como en otras.

Por el contrario, aunque el PD puede participar en algún grado de esa tendencia a experimentar ansiedad en múltiples situaciones (ansiedad rasgo o general), esto no parece ocurrir en la misma medida que en el SI, existiendo en aquél una mayor especificidad en cuanto a la experiencia de ansiedad, que estaría más circunscrita (o sería más intensa) a contextos que implican un componente de evaluación de la propia habilidad. Consistente con esta interpretación, nuestros datos ponían de manifiesto que la baja autoestima relacionada con aspectos académicos era más relevante en el PD que en el SI, mientras que en este último cobraba un mayor protagonismo una autoimagen negativa de carácter global.

La mayor presencia en el SI de una ansiedad general necesariamente conlle- 
va una mayor afectación de otros aspectos de la personalidad. Esto es lo que, al menos, puede deducirse de algunos resultados. Concretamente, los datos indicaban que la falta de claridad en el autoconcepto y la falta de autoeficacia generalizada predecían específicamente el SI, pero no el PD, y el externalismo presentaba asociaciones parciales mayores con el primero de ellos. Según esto, la mayor relevancia de la ansiedad rasgo en los impostores tiene repercusiones más amplias en la dinámica psicológica, ocasionando problemas en la identidad y en la percepción de competencia y capacidad para alterar el medio, algo que no parece estar presente en los pesimistas defensivos. En la base, al menos en parte, de este tipo de ansiedad experimentada por los sujetos impostores puede que esté su mayor tendencia a la externalización de los éxitos (Fernández y Bermúdez, en prensa), y, por tanto, al perfeccionismo y la autocrítica (Chrisman, et al., 1995; Kolligian y Sternberg, 1991), que, a su vez, implican la fijación de unas altos estándares de autoevaluación. Estos harían más probable la experiencia de ansiedad, pues ningún nivel de rendimiento en ningún contexto vital es reconocido por estos sujetos como suficiente o adecuado, lo que facilita el desarrollo de malestar emocional e insatisfacción personal crónicos.

La mayor relevancia de la ansiedad rasgo en el SI sugiere, asimismo, que este síndrome pueda predisponer a la experiencia de afecto negativo crónico. Esta idea sería coherente con lo defendido por Cozzarelli y Mayor (1990) y Kolligian (1990). Estos autores postulan que el fenómeno del impostor podría conceptualizarse como una manifestación específica de una tendencia más general a experimentar afectividad negativa, lo que implica una disposición a sufrir estados emocionales aversivos y a mantener una perspectiva negativa sobre el mundo
(Watson y Clark, 1984). No obstante, esta es una posibilidad que deberá ser contemplada más directamente por futuros estudios.

Por otra parte, en el PD es otra la dinámica psicológica que le define. Concretamente, en este constructo el aspecto esencial era el (bajo) optimismo y el pesimismo, contemplados como expectativas generalizadas sobre los resultados. El bajo optimismo era específico del PD, no del SI, tal y como indicaban las asociaciones relativas entre variables y los modelos predictivos hallados, y el pesimismo contribuía en mayor grado en el PD que en el SI. La cuestión que debemos plantearnos ahora es por qué estas variables personales resultan ser relevantes sólo o principalmente en el PD. Es muy probable que el carácter protector que proporcionan unas bajas expectativas de resultado a los pesimistas defensivos, pero no a los impostores, tenga algo que ver al respecto (véase Fernández y Bermúdez, en prensa, para un análisis detallado de esta cuestión). Concretamente, de la misma manera que un estilo atribucional desadaptativo justifica la fijación de unas bajas expectativas en una situación concreta, con valor protector (Blaine y Crocker, 1993; Fernández, 1999; Fernández y Bermúdez, en prensa), ese mismo estilo atribucioal puede traducirse también en una creencia generalizada de que es poco probable que ocurran resultados positivos en la vida (bajo optimismo) y de que es muy probable que ocurran resultados negativos (pesimismo). En última instancia, estos dos últimos tipos de expectativas permiten anticipar de manera crónica unos resultados negativos, lo que proporciona una preparación afectiva también permanente contra sus implicaciones, que es actualizada en cada nueva situación específica a afrontar, vía fijación de unas bajas expectativas específicas de resultado. 
Por otra parte, la consideración de un último grupo de variables permitirá establecer una nueva fuente de diferenciación entre el PD y el SI. Nos estamos refiriendo a los dos componentes de la autoconciencia, la privada y la pública, $y$ a lo que se considera como una reacción a esta última en un contexto social, la ansiedad social (Scheier y Carver, 1985b). Si bien habíamos especulado con la idea de que esta variable, la autoconciencia, pudiera ser relevante para ambos constructos, los datos se encargaron de demostrar que aquélla parece importante únicamente en el SI.

Como sugeríamos en un primer momento, la tendencia presente en el SI a desear evitạ la decepción y el rechazo de los demás mediante un análisis minucioso de la propia conducta y del efecto que ésta provoca en ellos (Fernández y Bermúdez, en prensa), requiere, necesariamente, una focalización en los aspectos privados y públicos de la propia conducta. En la medida en que en el PD no estaba presente esta necesidad de manejar las impresiones de los demás, se explicaría la ausencia de una asociación entre aquél y la autoconciencia en el presente estudio, tanto a partir de los análisis absolutos como de los relativos, en contra de lo informado por un estudio anterior (Fernández y Bermúdez, 1999). En este sentido, es muy posible que la asociación positiva moderada encontrada allí entre estas dos variables pueda ser debida a la presencia de sujetos pesimistas defensivos con altas puntuaciones en SI (en el citado estudio no se realizaron análisis relativos destinados a mantener constante el efecto de este último constructo).

Por otra parte, la ansiedad social también se revelaba a partir de los análisis relativos, como variable importante únicamente en el SI en consonancia con el poder explicativo que la autoconciencia pública ejercía sobre aquél. De manera congruente con esta tendencia a reaccionar con ansiedad en contextos sociales, la autoestima social también aportaba poder predictivo único y significativo en el caso del SI. La relevancia de lo social en este constructo es algo inherente a la conceptualización que normalmente se hace de él como un patrón de comportamiento orientado interpersonalmente (Clance, 1985; Fernández y Bermúdez, en prensa), dentro del cual resulta esencial la preocupación que muestran los sujetos impostores ante la posibilidad de que los demás puedan descubrir la falta de competencia que perciben en sí mismos, creencia que les impulsa, como ya señalamos, a presentar un patrón de autoobservación y sobrepreparación destinado a evitar un posible fracaso, que podría "delatarles», revelando así esa supuesta incompetencia. Este temor crónico a ser "descubiertos» explicaría en gran medida la ansiedad social que suelen experimentar, mientras que una baja autoestima social (y global) estaría en la base de estos temores y de las reacciones subsiguientes, en consonancia con lo sugerido por Langford y Clance (1993) y con lo encontrado por Cozzarelli y Major (1990).

Un último aspecto importante que es necesario tomar en consideración se refiere a la cuestión de la direccionalidad de los efectos descritos aquí. Nosotros hemos enfatizado continuamente el papel que distintas respuestas, creencias y actitudes (ansiedad, autoestima, autoconciencia, etc.) pueden tener en la instauración y mantenimiento del SI y del PD, cuando es perfectamente factible el efecto opuesto, esto es, que sean estos patrones de comportamiento los que influyan en aquellas otras variables. No obstante, como suele ser lo habitual en la manera de conceptualizar la dinámica de la personalidad, lo más probable es que exista una influencia recíproca entre ambos tipos de variables. 
Así, por ejemplo, una baja autoestima y una alta preocupación pueden contribuir al mantenimiento de sentimientos y reacciones de impostor, y éstos, a su vez, favorecer el mantenimiento de una baja autoestima y una alta preocupación. De acuerdo con la defensa de la existencia de un ciclo autoperpetuador de respuestas desadaptativas tanto en el SI (Langford y Clance, 1993) como en el PD (Fernández, 1999), hay que tener en cuenta que ambos patrones de comportamiento facilitan la consecución de importantes éxitos, que actuarían como reforzadores positivos de las creencias, actitudes y conductas que impostores y pesimistas defensivos mantienen previamente a la obtención de esos resultados. Estos sujetos terminarían por atribuir a aquéllas un papel catalizador en la obtención de los éxitos, que contribuirían a su mantenimiento, a pesar de los costos que la mayoría de ellas pueden acarrear.

\section{REFERENCIAS BIBLIOGRÁFICAS}

Blaine, B., y Crocker, J. (1993). Self-esteem and self-serving biases in reactions to positive and negative events: An integrative review. En R.F. Baumeister (Ed.), Self-esteem: The puzzle of low self-regard (pp. 55-81). New York: Plenum Press.

Campbell, J.D., Trapnell, P.D., Heine, S.J., Katz, I.M., Lavallee, L.F., y Lehman, D.R. (1996). Self-Concept Clarity: Measurement, personality correlates, and cultural boundaries. Journal of Personality and Social Psychology, 70, 141156.

Cantor, N., Norem, J.K., Niedenthal, P.M., Langston, C.A., y Brower, A.M. (1987). Life tasks, self-concept ideals and cognitive strategies in a life transition. Journal of Personality and Social Psychology, 53, 1178-1191.
Clance, P.R. (1985). The impostor phenomenon: When success makes you feel like a fake. Toronto: Bantam Books.

Clance, P.R., y Imes, S.A. (1978). The impostor phenomenon in high achieving women: Dynamics and therapeutic intervention. Psychoterapy: Theory, Research, and Practice, 15, 241-247.

Clance, P.R., y O'Toole, M.A. (1988). The impostor phenomenon: An internal barrier to empowerment and achievement. Women and Therapy, 6, 51-64.

Cozzarelli, C., y Major, B. (1990). Exploring the validity of the impostor phenomenon. Journal of Social and Clinical Psychology, 9, 401-417.

Craig, A.R., Franklin, J.A., y Andrews, G. (1984). A scales to measure locus of control of behaviour. British Journal of Medical Psychology, 57, 173-180.

Chrisman, S.M., Pieper, W.A., Clance, P.R., Holland, C.L., y Glickauf-Hughes, C. (1995). Validation of the Clance Impostor Phenomenon scale. Journal of Personality Assessment, 65, 456467.

Fernández, E. (1999). Variables personales y situacionales en la autorregulación en contextos de logro: el caso del optimismo, el pesimismo defensivo y la controlabilidad de la tarea. Tesis Doctoral (no publicada). Madrid: UNED.

Fernández, E., y Bermúdez, J. (1999a). Estructura factorial del Optimismo y el Pesimismo. Boletín de Psicología, 63, 7-26.

Fernández, E., y Bermúdez, J. (en prensa). Estilo atribucional, autoobservación y defensividad: el caso del síndrome del impostor y el pesimismo defensivo. Revista de Psicología Social.

Fleming, J.S., y Whalen, D.J. (1990). The personal and academic self-concept inventory: Factor structure and gender differences in high school and college samples. Educational and Psychological Measurement, 50, 957-967. 
Harlow, R.E., y Cantor, N. (1995). Overcoming a lack of self-assurance in an achievement domain: Creating agency in daily life. En M.H. Kernis (Ed.), Efficacy, agency, and self-esteem (pp. 170195). New York: Plenum Press.

Harvey, J.C. (1981). The impostor phenomenon and achievement: $A$ failure to internalize success. (Tesis doctoral, Temple University, 1981). Disertation Abstract International, 42, 4969B.

Hummer, M.K., Dember, W.N., Melton, R.S., y Schefft, B.K. (1992). On the partial independence of optimism and pessimism. Current Psychology: Research and Reviews, 11, 37-50.

Kolligian, J., Jr. (1990). Perceived fraudulence as a dimension of perceived incompetence. En R.J. Sternberg y J. Kolligian (Eds.), Competence considered (pp. 261-285). New Haven, CT: Yale University Press.

Kolligian, J., Jr., y Sternberg, R.J. (1991). Perceived Fraudulence in Young Adults: Is there an "impostor syndrome"? Journal of Personality Assessment, 56, 308-326.

Langford, J., y Clance, P.R. (1993). The impostor phenomenon: Recent research findings regarding dynamics, personality and family patterns and their implications for treatment. Psychoterapy, 30, 495-501.

Matthews, G., y Clance, P.R. (1985). Treatment of impostor phenomenon in psychotherapy clients. Psychoterapy in Private Practice, 3, 71-81.

Norem, J.K., e Illingworth, K.S. (1993). Strategy-dependent effects of reflecting on self and tasks: Some implications of optimism and defensive pessimism. Journal of Personality and Social Psychology, 65, 822-835.

Norem, J.K., y Cantor, N. (1986a). Anticipatory and post hoc cushioning strategies: Optimism and defensive pessimism in "risky" situations. Cognitive Therapy and Research, 10, 347-362.
Norem, J.K., y Cantor, N. (1986b). Defensive pessimism: "Harnessing" anxiety as motivation. Journal of Personality and social Psychology, 52, 1208-1217. Norem, J.K., y Cantor, N. (1990). Cognitive strategies, coping, and perceptions of competence. En R.J. Sternberg y J. Kolligian (Eds.), Competence considered (pp. 190-204). New Haven, CT: Yale University Press.

Rothbaum, F., Weisz, J.R., y Snyder, S.S. (1982). Changing the world and changing the self: A two process model of perceived control. Journal of Personality and Social Psychology, 42, 5-37. Scheier, M.F., y Carver, C.S. (1985a). Optimism, coping and health: Assessment and implications of generalized outcome expectancies. Health Psychology, 4, 219-247.

Scheier, M.F., y Carver, C.S. (1985b). The Self-Consciousness Scale: A revised version for the use with general populations. Journal of Applied Social Psychology, 15, 687-699.

Scheier, M.F., Carver, C.S., y Bridges, M.W. (1994). Distinguishing optimism from neuroticism (and trait anxiety, self-mastery, and self-esteem): A reevaluation of the Life Orientation Test. Journal of Personality and Social Psychology, 67, 1063-1078.

Sherer, M., Maddux, J.E., Mercandante, B., Prentice-Dunn, S., Jacobs, B., y Rogers, R.W. (1982). The Self-Efficacy Scale: construction and validation. Psychological Reports, 51, 663-671.

Showers, C., y Rubens, C. (1990). Distinguishing defensive pessimism from depression. Negative expectations and positive coping mechanisms. Cognitive Therapy and research, 14, 385-399.

Spielberger, C.D. (1980). Preliminary professional manual for the Test Anxiety Inventory. Palo Alto, CA: Consulting Psychologists Press.

Spielberger, C.D., Gorsuch, R.L., y Lus- 
hene, R.E (1970). State-Trait Inventory (STAI). Palo Alto CA: Consulting Psychologists Press. (Versión española: Cuestionario de Ansiedad Estado-Rasgo TEA Ediciones, Madrid, 1986).

Topping, M.E.H. (1983). The impostor phenomenon: A study of its construct validity and incidence in university faculty members. (Doctoral dissertation, University of South Florida, 1983). Dissertation Abstracts International, 44, 1948B-1949B.

Watson, D., y Clark, L.A. (1984). Negative affectivity: The disposition to experience aversive emotional states. Psychological Bulletin, 96, 465-490. 